\title{
Angiología
}

www.elsevier.es/angiologia

ORIGINAL

\section{Evaluación de la calidad de vida en pacientes con síndrome postrombótico}

\author{
J.A. González-Fajardo a,*, M. Martín-Pedrosaa ${ }^{a}$ L. Mengibar Fuente ${ }^{a}$, \\ R. Salvador Calvo ${ }^{a}$, A. Almaraz ${ }^{\mathrm{b}}$ y C. Vaquero ${ }^{a}$
}

aServicio de Angiología y Cirugía Vascular. Hospital Clínico Universitario de Valladolid. Valladolid. España.

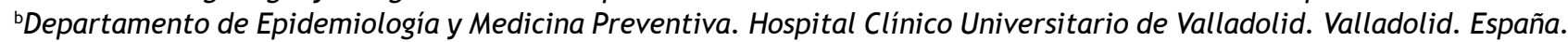

Recibido el 4 de mayo de 2010; aceptado el 26 de julio de 2010

\section{PALABRAS CLAVE \\ Trombosis venosa \\ profunda; \\ Calidad de vida; \\ Síndrome \\ postrombótico}

\begin{abstract}
Resumen
Introducción. Poco se conoce sobre el impacto del síndrome postrombótico (SPT) en la calidad de vida (CV) de los pacientes.

Objetivos. Evaluar la CV a largo plazo en pacientes con trombosis venosa profunda (TVP) de los miembros inferiores y examinar si ésta se correlaciona con el nivel de gravedad del SPT.

Material y métodos. Todos los pacientes incluidos fueron consecutivos, con síntomas unilaterales y primer episodio de TVP. Tras 5 años de seguimiento, el grado de secuela postrombótica fue valorado mediante la escala de Villalta. Al finalizar todos realizaron un cuestionario genérico (EuroQol) y otro específico de insuficiencia venosa (CIVIQ). Los resultados fueron analizados de manera ciega.

Resultados. De 165 pacientes inicialmente reclutados, tan sólo 100 completaron el seguimiento de 5 años y cumplimentaron los cuestionarios. A pesar del uso regular de medias elásticas (75\%), la incidencia de SPT fue del 65\%: 35 pacientes asintomáticos, 41 pacientes con SPT moderado y 24 pacientes con SPT grave. La CV media con el EuroQol y el CIVIQ fue, respectivamente, de $91,5 \%$ y $89,46 \%$ en SPT ausente; $85,9 \%$ y $74,43 \%$ en SPT moderado; $58,2 \%$ y $49,82 \%$ en SPT grave. El EuroQol tan sólo mostró diferencias significativas cuando el SPT fue grave, mientras que el CIVIQ se reveló muy útil para detectar diferencias entre los distintos subgrupos, mostrando un deterioro progresivo en relación con el nivel de gravedad del SPT $(r=0,601)$.

Conclusiones. Los pacientes con TVP que desarrollan SPT presentan un importante deterioro de la CV, tanto peor cuanto más grave sea la secuela postrombótica.

๔ 2010 SEACV. Publicado por Elsevier España, S.L. Todos los derechos reservados.
\end{abstract}

\footnotetext{
*Autor para correspondencia.

Correo electrónico: jafajardo@telefonica.net (J.A. González-Fajardo).
}

0003-3170/\$ - see front matter ๔ 2010 SEACV. Publicado por Elsevier España, S.L. Todos los derechos reservados. 


\section{KEY WORDS}

Deep venous

thrombosis;

Quality of life;

Posthrombotic

syndrome

\section{Quality of life after deep venous thrombosis}

\begin{abstract}
Introduction. Little is known about the impact of the posthrombotic syndrome (PTS) on the quality of life (QoL) of patients after deep vein thrombosis (DVT).

Objective. The aims of this study were to analyze the long-term QoL after DVT and to examine whether QoL correlates with the severity of PTS.

Material and methods. Patients with previous symptomatic, unilateral, and a first episode of DVT were enrolled in this study. After 5 years of follow-up, the venous disease was related to pathological severity of PTS according to the scale of Villalta. All patients then completed a generic (EuroQol) and a disease-specific one for venous insufficiency (CIVIQ) questionnaires. The analysis of results was blinded to clinical details of the patients.

Results. Of the 165 patients initially recruited, 100 patients completed the follow-up and completed the QoL assessment at 5 years. Despite the regular use of compression stockings the incidence of PTS was $65 \%$ : 35 asymptomatic, 41 with moderate-PTS, and 24 with severe-PTS. The mean QoL with the EuroQol and the CIVIQ was $91.5 \%$ and $89.46 \%$ in absent-PTS; $85.9 \%$ and $74.43 \%$ in moderate-PTS; and $58.2 \%$ and $49.82 \%$ in severe-PTS, respectively. The EuroQol only showed significant differences when the PTS was severe, while the CIVIQ was useful to detect differences among the different subgroups. These scores worsened significantly with increasing severity of PTS $(r=0.601)$.

Conclusions. Patients with DVT in whom PTS develops have a worse QoL than those without PTS. Patient-based QoL measures correlated well with the severity of PTS.

(c) 2010 SEACV. Published by Elsevier España, S.L. All rights reserved.
\end{abstract}

\section{Introducción}

Aunque en los últimos años se han realizado importantes progresos en el diagnóstico y tratamiento de la enfermedad tromboembólica venosa, poco se conoce sobre su impacto a largo plazo en la calidad de vida de estos pacientes ${ }^{1,2}$. El síndrome postrombótico (SPT) es una de las complicaciones más frecuentes después de una trombosis venosa profunda (TVP) en los miembros inferiores, pero su desarrollo es generalmente impredecible y la incidencia real desconocida porque se incrementa con el paso del tiempo ${ }^{3}$. No cabe duda de que aquellos pacientes que desarrollan signos y síntomas de insuficiencia venosa como consecuencia de la secuela postrombótica tendrán importantes limitaciones en su actividad física diaria, el desempeño del trabajo y sus relaciones sociales $^{4,5}$. Sin embargo, estas limitaciones no han sido adecuadamente cuantificadas, particularmente en nuestro país.

Durante los últimos años la calidad de vida ha emergido como un criterio mayor de salud ${ }^{6,7}$. Diversos tests genéricos y específicos de insuficiencia venosa han sido publicados en la literatura ${ }^{8-11}$, pero uno de los problemas más importantes ha sido la validación y reproducibilidad de estos cuestionarios y la traducción a diferentes idiomas, ya que la valoración de la calidad de vida difiere de unos países a otros según los ámbitos culturales ${ }^{12-14}$. En este sentido, el único test específico sobre insuficiencia venosa crónica validado en lengua española ha sido el $\mathrm{CIVIQ}^{15}$.

Recientemente, nuestro grupo publicó los resultados a largo plazo de un grupo bien definido de pacientes con TVP de los miembros inferiores ${ }^{16}$. Tras 5 años de seguimiento, la gravedad de la secuela postrombótica fue valorada mediante la escala de Villalta. El objetivo de este estudio ha sido evaluar la calidad de vida de estos pacientes mediante la aplicación de un test genérico (EuroQol) y un test específico de insuficiencia venosa (CIVIQ), y examinar si la calidad de vida se correlaciona con el nivel de gravedad del SPT.

\section{Material y métodos}

\section{Pacientes}

Se trata de una cohorte de pacientes históricos seguidos prospectivamente desde 1997 y en la que la prueba objetiva de confirmación diagnóstica era entonces la flebografía. En la fase inicial, fueron aleatorizados a un tratamiento anticoagulante con enoxaparina o dicumarina durante al menos tres meses ${ }^{17}$. Todos los pacientes incluidos en el presente estudio fueron consecutivos con síntomas unilaterales y con un primer episodio de TVP de los miembros inferiores. Los criterios de exclusión fueron: sospecha de tromboembolismo pulmonar (TEP), sangrado activo o anomalía de la coagulación que contraindicara la anticoagulación, episodios previos de TVP-TEP, colocación de filtro de vena cava, alergia a contrastes yodados o trombofilia conocida. Todos los pacientes fueron motivados a usar medias elásticas de compresión $(40 \mathrm{mmHg}$ en el tobillo) en sus actividades diarias durante al menos dos años.

\section{Criterio para síndrome postrombótico}

Después de 5 años de seguimiento, el grado de secuela postrombótica fue valorado de manera ciega por uno de los investigadores (MMP) mediante la escala validada de Villalta ${ }^{18}$. Esta escala evalúa 5 síntomas (dolor, calambres, pesadez, prurito y parestesias) y 6 signos (edema maleolar, induración de la piel, hiperpigmentación, nuevas ectasias venosas, eritema y dolor a la compresión de la pantorrilla) en relación con la insuficiencia venosa crónica. Para cada ítem se 
asigna una puntuación de 0 (no presente o mínimo) a 3 (grave). Una puntuación total mayor o igual a 15 o la presencia de una úlcera venosa indicó un SPT grave; una puntuación de 5 a 14 correspondió a un SPT moderado; y una puntuación menor de 5 se consideró normal o ausencia de SPT.

\section{Medición de la calidad de vida}

Todos los pacientes enrolados en este estudio completaron a los 5 años de seguimiento un cuestionario de calidad de vida genérico (EuroQol) y otro específico de insuficiencia venosa (CIVIQ). Estos cuestionarios fueron cumplimentados individualmente o con ayuda de un familiar o personal sanitario ajeno al estudio, dependiendo del grado de autonomía y nivel cultural de los participantes. Todos los resultados obtenidos fueron analizados de manera ciega, sin conocimiento del nivel de gravedad de la enfermedad venosa ni de las características demográficas o de tratamiento del paciente.

El EuroQol es un cuestionario genérico ampliamente utilizado en el mundo, que además de su fiabilidad y validez, permite ser realizado en tan sólo 2 minutos, lo que aporta una gran comodidad tanto para el paciente como para el médico $^{8,12}$. Este test valora 5 categorías: la movilidad, el cuidado personal, las actividades cotidianas, el dolor y el grado de ansiedad-depresión que la enfermedad provoca. Cada categoría se puntúa de 1 a 3 dependiendo del nivel de limitación, siendo 1 normal o ausente, 2 moderado y 3 cuando la repercusión es importante o muy importante. Por tanto, la puntuación total de la calidad de vida puede variar desde 5 (estado de salud satisfactorio) hasta 15 (deterioro máximo).

El CIVIQ es el único cuestionario específico de insuficiencia venosa validado en España ${ }^{15}$, que ha demostrado una excelente consistencia interna y una alta fiabilidad, y cuyo tiempo de realización no suele sobrepasar los 10 minutos. Se trata de un test con 20 ítems en el que cada pregunta tiene 5 opciones (valores de 1 a 5 ), de modo que la puntuación global mínima puede ser de 20 puntos (mejor estado de salud) y la máxima de 100 (deterioro importante). El valor obtenido es directamente proporcional al grado de deterioro de la calidad de vida, es decir, tanto mayor cuanto peor es el estado de salud. En este test se identifican 4 dimensiones: repercusiones psicológicas ( 9 ítems), repercusión del dolor (5 ítems), repercusión física ( 3 ítems) y repercusión social ( 3 ítems) $)^{9,13}$. La aplicación de este cuestionario permite, por tanto, calcular tres puntuaciones: una puntuación por cada ítem, una puntuación por dimensión y una puntuación global.

Para expresar los valores globales y de cada dimensión de 0 a 100 , los autores ${ }^{9}$ recomiendan la conversión matemática del valor obtenido según la siguiente fórmula: (puntuación final obtenida - puntuación mínima posible/puntuación teórica máxima - puntuación teórica mínima) $\times 100$. El porcentaje se invierte restándolo de 100 , de manera que 0 representa la peor calidad de vida y 100 la mejor.

Esta conversión matemática fue aplicada a los valores totales del EuroQol con objeto de permitir la comparación gráfica entre el test genérico y el específico.

\section{Análisis estadístico}

Los valores numéricos se expresaron como media \pm desviación típica o porcentaje (\%). El análisis se basó en la prue- ba $\mathrm{t}$ de Student para las diferencias de medias entre los diferentes grupos (SPT ausente, moderado y grave). Las medias de calidad de vida fueron ajustadas por edad y sexo usando el análisis de la varianza. La calidad de vida media se correlacionó con el nivel de gravedad del SPT mediante el coeficiente de correlación de Pearson. Se consideró significativa cualquier diferencia observada en los grupos que tuviera una probabilidad de ocurrencia menor de 0,05. Para valorar la consistencia interna del test específico se calculó el coeficiente $\alpha$ de Cronbach (coeficiente de correlación intraclase), considerándose valores superiores a 0,70 como aceptable. Todos los análisis se realizaron con el programa estadístico SPSS versión 14.0 (SPSS Inc, Chicago, IL, USA).

\section{Resultados}

De los 165 pacientes inicialmente incluidos en el estudio, tan sólo $100(60,6 \%)$ completaron el seguimiento a 5 años, registrándose 28 muertes y 37 pérdidas durante este periodo. Estos 100 pacientes realizaron los cuestionarios de calidad de vida sin que hubiera pérdida de datos. Esta población constituye nuestro grupo de trabajo y sus características clínicas aparecen registradas en la tabla 1.

Tabla 1 Características basales de los pacientes

\begin{tabular}{|c|c|}
\hline & Pacientes $(n=100)$ \\
\hline Edad (años) & $57,4 \pm 14,4$ \\
\hline Sexo: hombre/mujer & $50 / 50(50,0 \%)$ \\
\hline Peso $(\mathrm{kg})$ & $74,4 \pm 11,4$ \\
\hline Medias elásticas & $75(75,0 \%)$ \\
\hline \multicolumn{2}{|l|}{ Factores de riesgo para TVP } \\
\hline Trauma, cirugía o inmovilización & $26(26,0 \%)$ \\
\hline Cáncer (paraneoplásica) & $5(5,0 \%)$ \\
\hline Trombofilia & $9(9,0 \%)$ \\
\hline Idiopática & $40(40,0 \%)$ \\
\hline \multicolumn{2}{|l|}{ Localización del trombo } \\
\hline Venas gemelares con poplítea & $21(21,0 \%)$ \\
\hline Vena poplítea y femoral & $45(45,0 \%)$ \\
\hline Vena poplítea, femoral, e iliaca & $24(24,0 \%)$ \\
\hline Vena iliaca & $10(10,0 \%)$ \\
\hline \multicolumn{2}{|l|}{ Escala Marder (flebografía) } \\
\hline Antes del tratamiento & $21,7 \pm 11,2$ \\
\hline 3 meses después del tratamiento & $13,8 \pm 10,9$ \\
\hline \% regresión trombótica & $38,1 \pm 52,4$ \\
\hline \multicolumn{2}{|l|}{ Recurrencia tromboembólica } \\
\hline Total recurrencias (pacientes) & $27(27,0 \%)$ \\
\hline \multicolumn{2}{|l|}{ Recurrencia de TVP (eventos) } \\
\hline Ipsilateral & $23(23,0 \%)$ \\
\hline Contralateral & $6(6,0 \%)$ \\
\hline Embolismo pulmonar (eventos) & $4(4,0 \%)$ \\
\hline Hemorragia mayor & $1(1,0 \%)$ \\
\hline Síndrome postrombótico & $65(65 \%)$ \\
\hline Ausente & $35(35,0 \%)$ \\
\hline Moderado & $41(41,0 \%)$ \\
\hline Grave & $24(24,0 \%)$ \\
\hline
\end{tabular}

Datos expresados como media \pm desviación típica o porcentaje (\%). TVP: tromboembolismo pulmonar. 
A pesar del uso regular de medias elásticas de compresión (75\%), un total de 65 pacientes desarrollaron signos y síntomas de SPT. Éste fue considerado moderado en 41 pacientes y grave en 24. Tan sólo 35 pacientes fueron asintomáticos después de 5 años de seguimiento.

La aplicación del cuestionario CIVIQ mostró en nuestro estudio una alta consistencia interna, tanto global (valor $\alpha$ de Cronbach de 0,941 ) como para cada una de las dimensiones analizadas (tabla 2), lo que soporta la fiabilidad de este test para el estudio de pacientes con signos y síntomas de insuficiencia venosa crónica.

La calidad de vida total en relación con la gravedad de la secuela postrombótica fue con el EuroQol y con el $\mathrm{Cl}$ -

Tabla 2 Consistencia interna del cuestionario CIVIQ en nuestro estudio

\begin{tabular}{lcc}
\hline \multicolumn{1}{c}{ CIVIQ } & $\begin{array}{c}\text { Número } \\
\text { de ítems }\end{array}$ & $\begin{array}{c}\text { Coeficiente } \alpha \\
\text { de Cronbach }\end{array}$ \\
\hline Repercusión del dolor & 5 & 0,878 \\
Repercusión física & 3 & 0,880 \\
Repercusión social & 3 & 0,717 \\
Repercusión psicológica & 9 & 0,856 \\
Total & 20 & 0,941
\end{tabular}

VIQ, respectivamente, de $91,5 \%$ y $89,46 \%$ cuando el SPT estuvo ausente, de $85,9 \%$ y $74,43 \%$ cuando el SPT fue moderado, y de $58,2 \%$ y $49,82 \%$ cuando el SPT fue grave (tablas 3 y 4). Ajustada la calidad de vida por edad y sexo, no se observaron diferencias significativas para ninguna de estas variables. El EuroQol tan sólo demostró diferencias significativas cuando el deterioro fue grave, no siendo capaz de revelar diferencias en ninguna de las categorías valoradas (excepto el dolor) entre los pacientes con SPT ausente y SPT moderado. El CIVIQ, por el contrario, se mostró como un cuestionario eficaz para detectar diferencias entre los distintos subgrupos, mostrando un deterioro significativo de la calidad de vida en relación con la gravedad de la insuficiencia venosa (coeficiente de correlación $r=0,601$ ).

Con respecto a las diversas dimensiones analizadas, la aparición de un SPT parece no afectar a las repercusiones psicológicas hasta tanto el grado de deterioro es grave. Por el contrario, el dolor fue la categoría significativamente más deteriorada tanto con el test genérico como con el específico. Según el EuroQol, el SPT no afectó al cuidado personal de los pacientes y tan sólo cuando era grave alteró la movilidad, las actividades cotidianas o los estados de ansiedad o depresión. El CIVIQ, sin embargo, mostró una afectación importante en las repercusiones sociales y físicas desde estados moderados de la enfermedad.

Tabla 3 Resultados de calidad de vida según el cuestionario EuroQol

\begin{tabular}{lcccc}
\hline \multicolumn{1}{c}{ EuroQol } & SPT ausente (A) & SPT moderado (B) & SPT grave (C) & p \\
\hline Movilidad (1 ítem/3 ptos: 1-3) & $1,25 \pm 0,55$ & $1,33 \pm 0,51$ & $1,86 \pm 0,46$ & $a / b$ NS; a-b/c $<0,001$ \\
Cuidado personal (1 ítem/3 ptos: 1-3) & $1,20 \pm 0,52$ & $1,16 \pm 0,41$ & $1,36 \pm 0,49$ & $a / b$ NS; a-b/c NS \\
Actividades cotidianas (1 ítem/3 ptos: 1-3) & $1,25 \pm 0,55$ & $1,21 \pm 0,45$ & $1,64 \pm 0,58$ & $a / b ~ N S ; a-b / c<0,003$ \\
Dolor/malestar (1 ítem/3 ptos: 1-3) & $1,05 \pm 0,22$ & $1,53 \pm 0,57$ & $2,32 \pm 0,47$ & $<0,001$ \\
Ansiedad/depresión (1 ítem/3 ptos: 1-3) & $1,10 \pm 0,30$ & $1,26 \pm 0,55$ & $2,00 \pm 0,75$ & $a / b ~ N S ; a-b / c<0,001$ \\
Total (5 ítems/3 ptos: 5-15) & $5,85 \pm 1,66$ & $6,41 \pm 1,75$ & $9,18 \pm 2,12$ & $a / b ~ N S ; a-b / c p<0,001$ \\
Total* & $91,5 \%$ & $85,9 \%$ & $58,2 \%$ & $a / b ~ N S ; a-b / c p<0,001$ \\
\hline
\end{tabular}

Datos expresados como media \pm desviación típica.

*Media ajustada según la fórmula: (puntuación final - puntuación mínima posible/puntuación teórica máxima - puntuación teórica mínima $\times 100$. La puntuación obtenida se invierte $(100-$ puntuación), siendo 0 la peor calidad de vida y 100 la mejor.

NS: no significativo; SPT: síndrome postrombótico.

Tabla 4 Resultados de calidad de vida según el cuestionario CIVIQ

\begin{tabular}{lcccc}
\hline \multicolumn{1}{c}{ CIVIQ } & SPT ausente (A) & SPT moderado (B) & SPT grave (C) & $\mathrm{p}$ \\
\hline R. psicológica (9 ítems/5 ptos: 9-45) & $14,80 \pm 7,02$ & $17,75 \pm 7,09$ & $24,95 \pm 6,86$ & $\mathrm{a} / \mathrm{b}$ NS; a-b/c p $<0,001$ \\
R. dolor (5 ítems/5 ptos: 5-25) & $6,95 \pm 1,82$ & $10,20 \pm 3,93$ & $15,95 \pm 3,89$ & $\mathrm{p}<0,001$ \\
R. físicas (3 ítems/5 ptos: 3-15) & $4,10 \pm 2,88$ & $7,07 \pm 3,99$ & $10,22 \pm 2,74$ & $\mathrm{p}<0,001$ \\
R. sociales (3 ítems/5 ptos: 3-15) & $4,00 \pm 1,48$ & $6,35 \pm 3,03$ & $9,00 \pm 2,92$ & $\mathrm{p}<0,001$ \\
Total (20 ítems/5 ptos: 20-100) & $28,43 \pm 10,28$ & $40,45 \pm 15,98$ & $60,14 \pm 13,02$ & $\mathrm{p}<0,001$ \\
Total* $^{*}$ & $89,46 \%$ & $74,43 \%$ & $49,82 \%$ & $\mathrm{p}<0,001$ \\
\hline
\end{tabular}

Datos expresados como media \pm desviación típica.

*Media ajustada según la fórmula: (puntuación final - puntuación mínima posible/puntuación teórica máxima - puntuación teórica mínima) $\times 100$. La puntuación obtenida se invierte $(100-$ puntuación), siendo 0 la peor calidad de vida y 100 la mejor.

NS: no significativo; R.: respuesta; SPT: síndrome postrombótico. 


\section{Discusión}

A pesar de un correcto tratamiento de la TVP y el uso regular de medias elásticas, la incidencia de SPT sigue siendo elevada; de hecho, un $65 \%$ de los pacientes de nuestro estudio mostró a los 5 años algún grado de insuficiencia venosa, según la escala validada de Villalta. Estos pacientes presentaron a largo plazo una calidad de vida peor que aquellos que no la desarrollaron, lo que subraya la importancia de la prevención y el tratamiento de esta complicación. Este estudio es uno de los primeros trabajos en España que cuantifica a largo plazo el grado de deterioro que la secuela postrombótica produce en la calidad de vida ${ }^{19}$, demostrando la presencia de una correlación según la cual cuanto más grave es el SPT tanto peor es la calidad de vida que el paciente presenta. De acuerdo con nuestros resultados, el dolor es la dimensión significativamente más afectada en los pacientes con SPT. Este malestar se puso de manifiesto entre los distintos subgrupos (ausente, moderado, grave) y fue detectado tanto por el EuroQol como por el CIVIQ. Por el contrario, las repercusiones psicológicas parecen ser las menos relevantes, de hecho tan sólo se presentan cuando los pacientes tienen un SPT grave. Una limitación, sin embargo, importante es el elevado número de pérdidas durante el seguimiento (40\%), lo que puede devaluar la potencia de este estudio.

Hasta ahora muy pocos estudios han valorado la calidad de vida a largo plazo después de padecer una TVP1,7. Estos trabajos señalaron el detrimento importante en la percepción de la salud de estos pacientes, a pesar del empleo de definiciones no homogéneas de SPT y el uso de instrumentos no estandarizados para la cuantificación objetiva del deterioro. En este sentido, Khan et $\mathrm{al}^{2,4,5}$ señalaron la importancia de utilizar cuestionarios específicos de insuficiencia venosa. En un estudio de 41 pacientes con un $46 \%$ de incidencia de SPT, tan sólo los tests específicos (VEINES-QOL y VEINES-Sym) fueron capaces de mostrar el impacto de la enfermedad en la calidad de vida, ya que el empleo de un test genérico como el SF-36 no detectó diferencias entre los grupos. Por tanto, aunque para la valoración global de la calidad de vida puedan ser necesarios cuestionarios genéricos que permiten evaluar cualquier condición médica y generalizar los resultados com- parándolos con otras patologías ${ }^{20,21}$, este tipo de cuestionarios presenta importantes limitaciones. De hecho, en nuestro estudio la aplicación del EuroQol fue útil tan sólo para objetivar y medir el impacto de la insuficiencia venosa cuando el grado de deterioro fue grave, siendo incapaz de discernir entre grados moderados o ausentes de enfermedad.

De los 7 cuestionarios específicos desarrollados para valorar la calidad de vida en pacientes con insuficiencia venosa crónica ${ }^{11}$, tan sólo el CIVIQ ha sido validado en lengua española. Este cuestionario fue inicialmente elaborado por Launois ${ }^{9}$ en Francia y su validación psicométrica fue realizada en España por Lozano et al $^{15}$ en una muestra de 476 pacientes del estudio RELIEF. La aplicación de este test en nuestro trabajo nos ha permitido detectar diferencias entre los distintos subgrupos, demostrando así su mayor validez discriminante para valorar este tipo de patologías. Los hallazgos de alta consistencia interna e importante coeficiente de correlación señalan que la reproducibilidad es óptima para objetivar y medir la calidad de vida en pacientes con insuficiencia venosa crónica en nuestro país.

La trascendencia clínica de este estudio es que nos permite por primera vez comparar la calidad de vida global de un paciente con SPT grave $(58,20 \%)$ con respecto a otras patologías médicas mediante un instrumento de cuantificación objetiva del deterioro (EuroQol índice medio). Aun reconociendo las posibles limitaciones, podemos observar que tan sólo la insuficiencia cardíaca grave (51\%) y el ángor inestable $(45 \%)$ muestran valores inferiores. Tanto la enfermedad pulmonar obstructiva crónica (61\%), el sida sin complicaciones $(70 \%)$, la diabetes mellitus tipo $2(69 \%)$ y la diabetes mellitus tipo 1 (62\%) muestran una mejor calidad de vida (fig. 1). Si esta comparación la hacemos con diversos procesos neoplásicos, podemos apreciar que salvo el cáncer de esófago (42\%), el cáncer de pulmón (58\%) y los procesos oncológicos generales tras 4 meses de tratamiento (54\%), el resto de neoplasias presenta un detrimento de la calidad de vida menor que un SPT grave (fig. 2). Estos datos muestran el enorme impacto que un SPT grave tiene en la percepción de salud de los pacientes y justifica la importancia de la prevención de esta complicación en aquellos pacientes que presentan una enfermedad tromboembólica venosa.

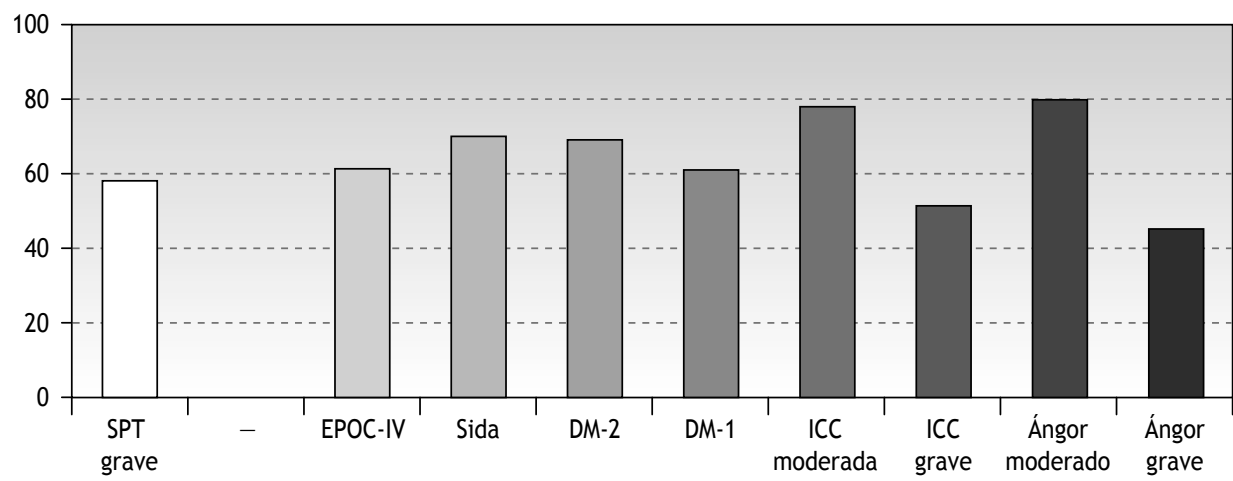

EuroQol

Figura 1 Comparación gráfica de la calidad de vida global (EuroQol índice medio) del síndrome postrombótico (SPT) grave con diversas patologías. El valor medio de la calidad de vida se ha estimado según Dyer et al $^{20}$, y la puntuación total ha sido ajustada, con 0 representando detrimento máximo y 100 la mejor calidad de vida posible. DM: diabetes mellitus; EPOC: enfermedad pulmonar obstructiva crónica; ICC: insuficiencia cardíaca congestiva. 


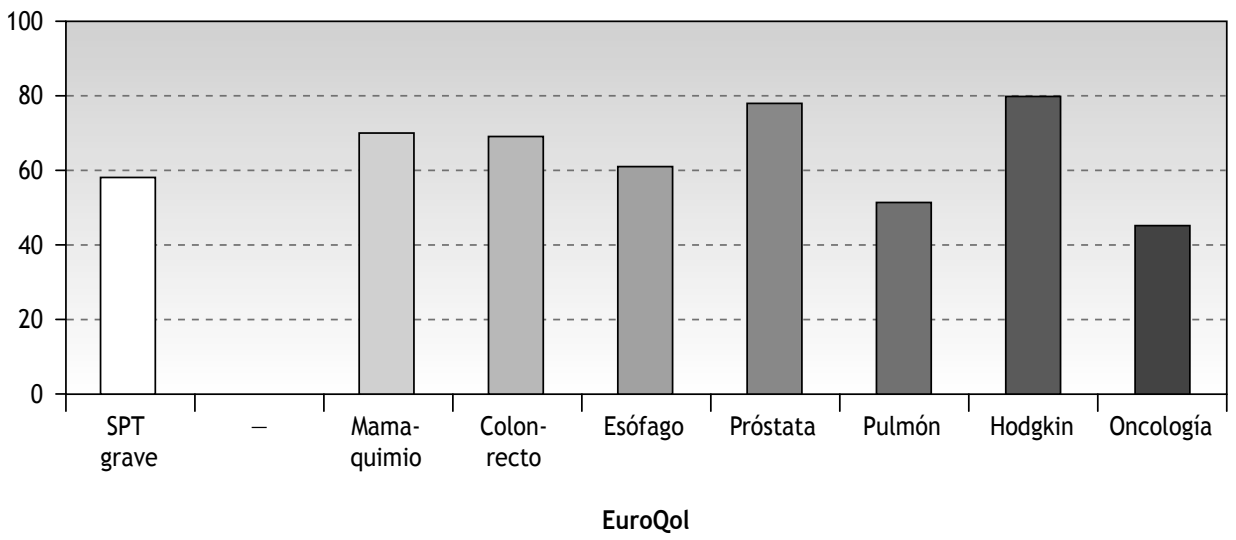

Figura 2 Comparación gráfica de la calidad de vida global (EuroQol índice medio) del síndrome postrombótico (SPT) grave con diversas neoplasias. El valor medio de la calidad de vida se ha estimado según Pickard et al ${ }^{21}$, y la puntuación total ha sido ajustada, con 0 representando detrimento máximo y 100 la mejor calidad de vida posible. Debe tenerse en cuenta que la calidad de vida en las distintas neoplasias varía según su estadio, periodo evolutivo (prequirúrgico o posquirúrgico) y medidas adyuvantes asociadas (quimioterapia y/o radioterapia).

En conclusión, los pacientes con TVP en los miembros inferiores que desarrollan un SPT presentan un importante deterioro en su calidad de vida, tanto peor cuanto más grave sea la secuela postrombótica. La aplicación de un cuestionario específico, como el CIVIQ, permite detectar diferencias entre los distintos subgrupos, indicando que en nuestro medio el dolor es la dimensión más afectada y la repercusión psicológica la menos relevante.

\section{Conflicto de intereses}

Los autores declaran no tener ningún conflicto de intereses.

\section{Bibliografía}

1. Beyth RJ, Cohen AM, Landefeld CS. Long-term outcomes of deep vein thrombosis. Arch Intern Med. 1995;155:1031-7.

2. Khan SR, Hirsch A, Shrier I. Effect of postthrombotric syndrome on health-related quality of life after deep venous thrombosis. Arch Intern Med. 2002;162:1144-8.

3. Prandoni P, Lensing AW, Cogo A, Cuppini S, Villalta S, Carta M, et al. The long-term clinical course of acute deep venous thrombosis. Ann Intern Med. 1996;125:1-7.

4. Khan SR, M'Lan CE, Lamping DL, Kurz X, Berards A, Abenhaim L; for the Veines Study Group. The influence of venous thromboembolism on quality of life and severity of chronic venous disease. J Thromb Haemost. 2004;2:2146-51.

5. Kahn SR, Ducruet T, Lamping DL, Arsenault L, Miron MJ, Roussin $A$, et al. Prospective evaluation of health-related quality of life in patients with deep venous thrombosis. Arch Intern Med. 2005; $165: 1173-8$.

6. Price P, Harding K. Quality of life (letter to the editor). Lancet. 1995;346:445.

7. Lamping DL. Measuring health-related quality of life in venous disease: practical and scientific considerations. Angiology. 1997;48:51-7.

8. Brooks R. EuroQol: the current state of play. Health Policy. 1996;37:53-72.

9. Launois R, Reboul-Marty J, Henry B. Construction and validation of a quality of life questionnaire in Chronic Lower Limb Venous Insufficiency (CIVIQ). Qual Life Res. 1996;5:539-54.
10. Kahn SR, M'Lan CE, Lamping DL, Kurz X, Berards A, Abenhaim L, and VEINES Study Group. Relationship between clinical classification of chronic venous disease and patient reported quality of life: results from an international cohort study. J Vasc Surg. 2004;39:823-8.

11. Lozano FS, Areitio-Aurtena A. Calidad de vida y enfermedad tromboembólica venosa. Med Clin (Barc). 2008;131 Supl 2: 37-41.

12. Badia X, Roset M, Montserrat S, Herdman M, Segura A. La versión española del euroQol: descripción y aplicaciones. Med Clin (Barc). 1999;112 Supl 1:79-86.

13. Jantet G. Chronic venous insufficiency: worldwide results of the RELIEF study. Angiology. 2002;53:245-56.

14. Erevnidou K, Launois R, Katsamouris A, Lionis C. Translation and validation of a quality of life questionnaire for chronic lower limb venous insufficiency into greek. Int Angiol. 2004;23:394-9.

15. Lozano FS, Launois R, and RELIEF Spanish group. Quality of Life (Spain and France): Validation of the Chronic Venous Insufficiency Questionnaire (CIVIQ). Methods Find Exp Clin Pharmacol. 2002;24:425-9.

16. González-Fajardo JA, Martín-Pedrosa M, Castrodeza J, Tamames $S$, Vaquero-Puerta $C$. Effect of the anticoagulant therapy in the incidence of post-thrombotic syndrome and recurrent thromboembolism: comparative study of enoxaparin versus coumarin. J Vasc Surg. 2008;48:953-9.

17. González-Fajardo JA, Arreba E, Castrodeza J, Pérez JL, Fernández L, Agúndez I, et al. Venographic comparison of subcutaneous low-molecular weight heparin with oral anticoagulant therapy in the long-term treatment of deep venous trombosis. J Vasc Surg. 1999;30:283-92.

18. Villalta S, Bagatella P, Piccioli A, Lensing AW, Prins MH, Prandoni $P$. Assessment of validity and reproducibility of a clinical scale for the PTS. Haemostasis. 1994;24:158A.

19. Bellmunt-Montoya S, González-Cañas E, Solanich-Valldaura T, Hospedales-Salomó J, Lloret-Cano MD, Vallespin-Aguado J, et al. ¿Qué le depara al paciente tras el diagnóstico de TVP? Estudio de factores pronósticos de la mortalidad, síndrome postrombótico y calidad de vida. Angiología. 2006;58:39-49.

20. Dyer MT, Goldsmith KA, Sharples LS, Buxton MJ. A review of health utilities using the EQ-5D in studies of cardiovascular disease. Health Qual Life Outcomes. 2010;8:13.

21. Pickard AS, Wilke CT, Lin HW, Lloyd A. Health utilities using the EQ-5D in studies of cancer. Pharmacoeconomics. 2007;25:365-84 